\title{
Herramienta en realidad virtual para el aprendizaje del lenguaje de señas mexicano
}

\author{
Arturo Borja Araiza, David Araujo Díaz, Laura Méndez Segundo \\ Instituto Politécnico Nacional, Escuela Superior de Cómputo, \\ Ciudad de México, México \\ aborja.isc@gmail.com, daraujo@ipn.mx \\ lmendezs.escomegmail.com
}

\begin{abstract}
Resumen. Este trabajo brinda una herramienta visual que permite el aprendizaje de múltiples palabras que son parte del lenguaje de señas mexicano (LSM) a través de un modelo antropomórfico completamente manipulable y programable, haciendo uso de los recursos brindados por la tecnología de realidad virtual utilizando el lenguaje VRML (Virtual Reality Modeling Language) y utilizando una base de datos creada a partir de los movimientos y posiciones bien establecidos por el LSM, representando distintos gestos y expresiones manuales, desde distintos ángulos, profundidades y a diversas velocidades, según lo desee y requiera el usuario, para optimizar el aprendizaje de dicho lenguaje utilizando una interfaz gráfica amigable.
\end{abstract}

Palabras clave: realidad virtual, lenguaje de señas, aprendizaje, aplicación, interacción humano-computadora.

\section{Virtual Reality Tool for Learning Mexican Sign Language}

\begin{abstract}
This work provides a visual tool for learning multiple words that are part of Mexican Sign Language (LSM) through a fully manipulable and programmable anthropomorphic model, using resources provided by the technology of virtual reality using the language VRML (Virtual Reality Modeling Language) and using a database created from the movements and positions well established by LSM, representing different gestures and manual expressions, from different angles, depths at various speeds, as desired and required by the user, to optimize the learning of said language using a friendly graphical interface.
\end{abstract}

Keywords: virtual reality, language, learning, application, human-computer Interaction. 


\section{Introducción}

El lenguaje es el medio de acceso humano al mundo. Los lenguajes tienen la virtud de abrirnos formas alternativas de pensar y entender el lugar que habitamos, relacionarnos con él, ampliarlo y modificarlo. Como posibilidad de comunicación, los lenguajes nos abren oportunidades para relacionarnos con otras personas, acercarnos a ellas y desarrollar una comprensión más amplia de ellas, de lo social y lo humano [1].

Aprender un lenguaje es acceder de un modo distinto al mundo que todas y todos conformamos, forma parte de la diversidad, los ideales, los conceptos mediante los cuales nos reconocemos, nos relacionamos y en último de los casos nos reinventamos.

El lenguaje tiene la virtud de permitirnos transformar las concepciones que tenemos de nosotros mismos y de las y los demás. Las personas nos conocemos fundamentalmente por medio de procesos comunicativos; por ello, entre más amplios sean éstos más posibilidades tenemos de diversificar nuestro mundo.

Sin embargo, hay muchas personas con problemas para comunicarse, siendo la sordera una de las más comunes. Las personas con este problema hacen uso del Lenguaje de Señas (LS) para poder comunicarse e incluso se han desarrollado sistemas de traducción (Voz/Texto-a-LS) para asistir en esta tarea. Sin embargo, debido a que los LS son dependientes de países y culturas, hay diferencias entre gramáticas, vocabularios y señas, incluso si estos provienen de lugares con lenguajes hablados similares.

\subsection{Estado del arte}

En 2003, la lengua de señas mexicana se declaró oficialmente una "lengua nacional", junto con las lenguas indígenas y el español, que se utiliza en el sistema nacional de educación para sordos. Antes de eso la principal filosofía educativa en el país se centró en el oralismo (voz y lectura de labios) y con pocas escuelas donde las clases se impartieran en el lenguaje de señas mexicano (LSM) [2].

En el 2018 se estimó que alrededor de 694,451 habitantes del país padecen deficiencia auditiva, de acuerdo con cifras del Censo de Población y Vivienda más reciente, realizado por el Instituto Nacional de Estadística y Geografía (INEGI) [3].

Debido a la falta de interés por parte de los especialistas en sistemas en abordar el LSM no se encontraron programas o sistemas similares que cubran la necesidad del aprendizaje del LSM, pues existen algunos sistemas similares que buscan el aprendizaje por medio de un videojuego[6] o reconocimiento visual de gestos humanos[7], pero están centrados en su mayoría para el lenguaje de señas americano, por lo que se propone este trabajo para cubrir las necesidades de este sector, y se propone el uso de la realidad virtual para dar soporte a las personas interesadas en aprender el LSM.

Recientemente se ha incrementado el número de personas que, sin padecer deficiencias auditivas, busca aprender el LSM para poder comunicarse con este grupo.

\subsection{Realidad virtual}

La Realidad Virtual (VR) es una representación de la realidad a través de medios electrónicos, que nos da la sensación de experimentar una situación real, en la que podemos interactuar con lo que nos rodea [4]. 
En este caso se trabajó con la VR no inmersiva, también conocida como realidad virtual de escritorio, que se limita al uso de una computadora y no requiere el uso de otros dispositivos como cascos o visores de VR; Debido a su bajo costo este tipo de realidad virtual ha sido ampliamente difundida y aceptada.

\subsection{Metodología}

Modelo de Desarrollo Rápido de Aplicaciones. El desarrollo rápido de aplicaciones o RAD por sus siglas en ingles Rapid Application Development., es un proceso de desarrollo de software, desarrollado inicialmente por James Martin en 1980. El método comprende el desarrollo iterativo, la construcción de prototipos y el uso de utilidades CASE. Tradicionalmente, el desarrollo rápido de aplicaciones tiende a englobar también la usabilidad, utilidad y la rapidez de ejecución.

Si se comprenden bien los requisitos y se limita el ámbito del proyecto, el proceso permite al equipo de desarrollo crear un "sistema completamente funcional" dentro de periodos cortos de tiempo. Cuando se utiliza principalmente para aplicaciones de sistemas de información, el enfoque comprende las siguientes fases:

Modelado de gestión. El flujo de información entre las funciones de gestión se modela de forma que responda a las siguientes preguntas: ¿Qué información conduce el proceso de gestión? ¿Qué información se genera? ¿Quién la genera? ¿A dónde va la información? ¿Quién la proceso?

Modelado de datos. El flujo de información definido como parte de la fase de modelado de gestión se refina como un conjunto de objetos de datos necesarios para apoyar la empresa. Se definen las características (llamadas atributos) de cada uno de los objetos y las relaciones entre estos objetos.

Modelado de proceso. Los objetos de datos definidos en la fase de modelado de datos quedan transformados para lograr el flujo de información necesario para implementar una función de gestión. Las descripciones del proceso se crean para añadir, modificar, suprimir, o recuperar un objeto de datos. Es la comunicación entre los objetos.

Generación de aplicaciones. El DRA asume la utilización de técnicas de cuarta generación. En lugar de crear software con lenguajes de programación de tercera generación, el proceso DRA trabaja para volver a utilizar componentes de programas ya existentes (cuando es posible) o a crear componentes reutilizables (cuando sea necesario). En todos los casos se utilizan herramientas automáticas para facilitar la construcción del software.

Pruebas de entrega. Como el proceso DRA enfatiza la reutilización, ya se han comprobado muchos de los componentes de los programas. Esto reduce tiempo de pruebas. Sin embargo, se deben probar todos los componentes nuevos y se deben ejercitar todas las interfaces a fondo.

\section{Diseño}

El diseño de esta herramienta consiste en un modelo de VR para imitar los movimientos hechos con el cuerpo hechos por las personas con discapacidad auditiva para comunicarse. 
Entre las acciones necesarias para llevar a cabo este proyecto fue necesario conocer y realizar los movimientos en VR de algunas palabras del LSM para probar la funcionalidad del modelo, así como programar los interpoladores de tiempo y posición, para posteriormente relacionarlos con los sensores de toque, para las funciones de reproducción, avance, retroceso, retardo y pausa de la animación del modelo (Fig. 1).

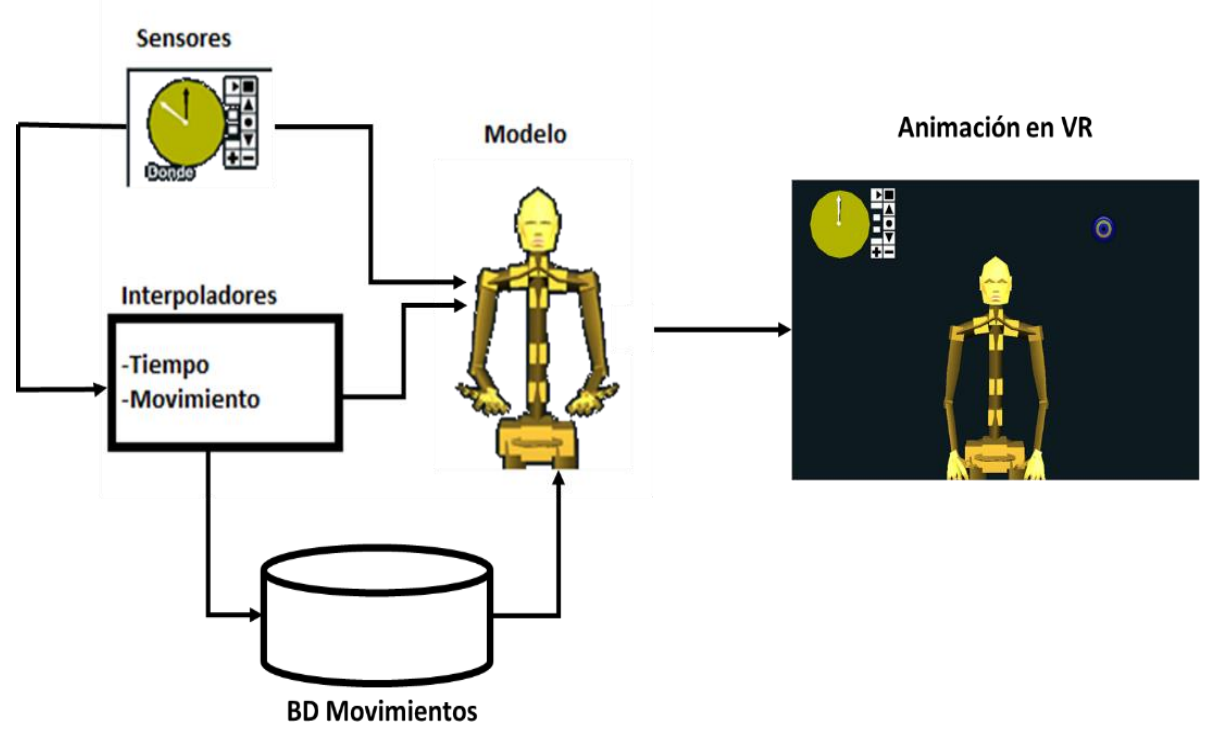

Fig.1. Diseño de la herramienta.

\section{Desarrollo}

La presente herramienta en Realidad Virtual (VR) para el aprendizaje del Lenguaje de Señas Mexicano (LSM) tiene como objetivo permitir a cualquier persona interesada, aprender diversas palabras del LSM, a través de un medio visual y permitiendo la manipulación de diferentes aspectos de la animación, como son, la velocidad de los gestos, su sentido, las palabras que se representarán, así como el poder pausar y reanudar la animación, entre otras.

Por ejemplo, para aprender correctamente los movimientos necesarios para representar la palabra "Rojo" podemos observar que se debe llevar el índice a la barbilla y realizar un movimiento vertical sobre esta (Fig.2), y para representar un vaso se hace el ademán de tomar un vaso o copa con una mano sobre la otra (Fig. 3).

La animación en paralelo, nos permite ver conjuntamente la palabra que se representa y la animación del modelo humano como se observa en la Fig. 4, asi como manipular la animación a través de los controles superiores (Fig. 5) con los cuales podremos manipular la velocidad a la que el modelo hace los gestos, que gesto es el que queremos ver, pausar la animación e invertir el sentido de las palabras; esto debido a que muchas veces difiere la estructura de una oración en contraste con su representación en LSM. 
Herramienta en realidad virtual para el aprendizaje del lenguaje de señas mexicano

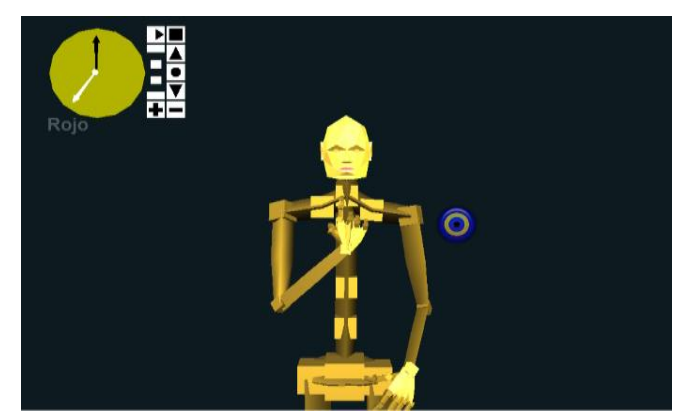

Fig. 2. Modelo diciendo ROJO. Elaboración Propia.

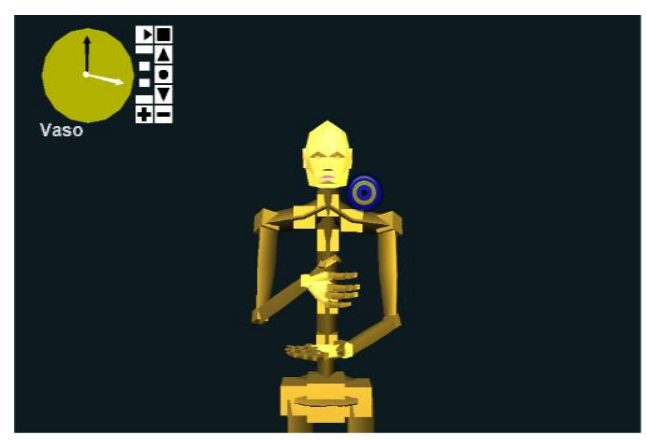

Fig. 3. Representación de un vaso en el LSM. Elaboración Propia.

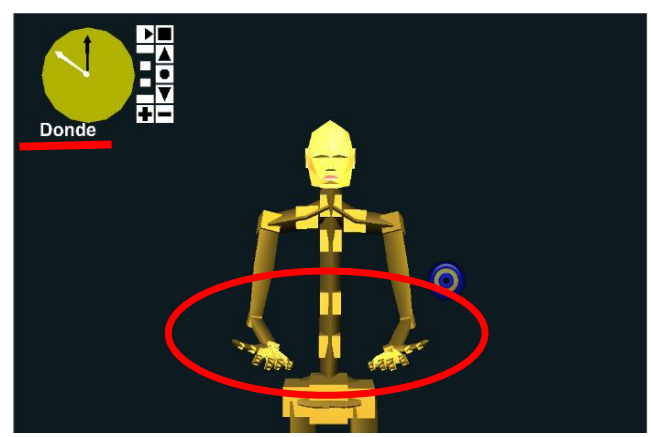

Fig. 4. Funcionamiento paralelo. Elaboración Propia.

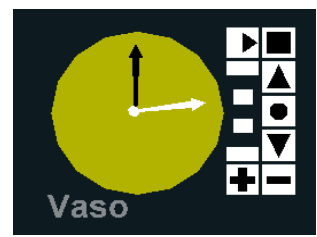

Fig. 5. Controles y timer de la aplicación. Elaboración Propia. 
El desarrollo de esta herramienta se realizó utilizando el lenguaje VRML (Virtual Reality Modeling Language) [4], debido a la sencillez y la facilidad que este lenguaje ofrece para representar mundos virtuales con recursos computacionales reducidos.

También se utilizó una base de datos propia, creada a partir de los movimientos y posiciones bien establecidos por el LSM, se basó principalmente en el diccionario del Consejo Nacional para Prevenir la Discriminación publicado en 2011 [2] y que se puede consultar en la red.

Para quien tenga el deseo de aprender, practicar y comprender el LSM, esta herramienta resulta una alternativa didáctica, interactiva, dinámica y amigable al usuario, ya que permite aprender y trabajar a un ritmo propio [5].

\section{Trabajo a futuro}

Para ampliar el presente trabajo se propone implementar una base de datos más extensa que permita crear un compendio completo de las palabras y expresiones que posee el LSM, y posteriormente aplicarlo a otros LS de alguna región o cultura diferente, así como evaluar si utilizando este método se presenta alguna mejora significativa en el aprendizaje en comparación con el método estándar de enseñanza de este lenguaje.

Cuando el sistema contenga una base de datos más completa, se evaluará su usabilidad que es un criterio importante en el rendimiento y calidad del sistema de RV, y aunque su evaluación puede tener un componente subjetivo, el estudio de normas, guías, estándares y diferentes propuestas permitirá unificar los aspectos relevantes en este proceso y servir como base para estructurar una guía que permita mejorar el sistema de LSM.

Existen múltiples campos del cómputo actual que podrían aprovechar herramientas como esta para desarrollar programas o traductores para gente con discapacidad auditiva.

\section{Conclusiones}

El prototipo desarrollado es un prototipo que conjuga la tecnología y herramientas existentes en la actualidad, para ayudar al aprendizaje de un lenguaje nuevo, mediante el uso de modelos virtuales. Este tipo de herramientas podrían servir para cerrar la brecha que existe en cuanto a la comunicación se refiere, permitiendo que cualquiera pueda acceder o utilizar las nuevas tecnologías y herramientas, así como ser aprovechado para implementarse con sistemas de inteligencia artificial [8] o deep learning [9] que puedan ser operados por medio de gestos del LSM.

Se considera que el uso de la realidad virtual permite mejorar el aprendizaje de un nuevo lenguaje, pues a diferencia de un video o un conjunto de imágenes, la interacción con el ambiente virtual es mayor, debido a que en el Sistema de RV propuesto se tienen controles de velocidad, lo que hace posible ver los gestos la cantidad de veces necesarias y controlando el tiempo de que estos se reproducen. 


\section{Referencias}

1. García Mejía, J.F., Tinoco Monroy, L.A. (et. al.): Modelado e implementación de un sistema de enseñanza de Lenguaje de Señas Mexicano. CIENCIA ergo-sum 14(2), 185-190 (2007)

2. Serafín de Fleischmann, M.E., González Pérez, R.: Manos con voz: Diccionario de lenguaje de señas mexicano. Consejo Nacional para Prevenir la Discriminación, México (2011)

3. Portal INEGI, https://www.inegi.org.mx/temas/discapacidad/default.html. Último acceso: $02 / 04 / 2019$

4. Araujo Diaz, D.: El Placer de Programar con VRML 2.0, Instituto Politécnico Nacional, México (2006)

5. Báez, M.: Las TIC: oportunidades para la alfabetización de jóvenes y adultos sordos. Centro de Estudios Interdisciplinarios de la Universidad Nacional de Rosario (CEI/UNR) Rosario, Argentina (2012)

6. Gameiro, J., Cardoso, T., Rybarczyk, Y. (eds): Innovative and Creative Developments in Multimodal Interaction Systems. Kinect-Sign: Teaching Sign Language to "Listeners" through a Game (2014)

7. Cooper, H., Holt, B., Bowden, R.: Sign Language Recognition. Visual Analysis of Humans, pp. 539-562. Springer (2011)

8. Stoll, S., Camgöz, N.C., Hadfield, S., Bowden, R.: Sign Language Production using Neural Machine Translation and Generative Adversarial Networks. In: 29th British Machine Vision, Northumbria University, Newcastle Upon Tyne, UK (2018)

9. Chung, S., Lim, J.Y., Ju Noh, K., Kim, G., Jeong, H.: Sensor Data Acquisition and Multimodal Sensor Fusion for Human Activity Recognition Using Deep Learning (2019) 